\title{
The Stomatological Complications of Diamond-Blackfan Anemia: A Case Report
}

\author{
Rita Fabiane Teixeira Gomes and Maria Cristina Munerato, PhD
}

\begin{abstract}
Diamond-Blackfan Anemia (DBA) is a rare heterogeneous genetic disease characterized by severe anemia, reduction or absence of erythroid progenitors, and pro-apoptoptic hematopoiesis, which culminates in bone marrow failure. The disease generally manifests in infancy, as craniofacial, cardiac, genitourinary, and upper limb congenital anomalies. Therapy with corticoids is the treatment of choice, while blood transfusion is adopted during diagnosis and as a chronic approach if the patient does not respond to corticoids. This case report describes DBA in a patient that presented with lesions on the oral mucosa caused by secondary neutropenia. The stomatologist plays an important role in a transdisciplinary team and must remain attentive to the general health conditions of patients, since some oral lesions may be associated with systemic events.
\end{abstract}

Keywords: Diamond-Blackfan Anemia; Blood transfusion; Delayed diagnosis; Neutropenia; Oral manifestations; Steroids

$\mathrm{D}$ iamond-Blackfan Anemia (DBA) is a rare, heterogeneous, genetic disease characterized by severe anemia, reduced counts or absence of erythroid progenitors, and pro-apoptoptic hematopoiesis, which culminates in bone marrow failure. ${ }^{1-5}$ The etiology of DBA is unknown, and though the disease usually manifests in infancy, ${ }^{1,6-14}$ it is not restricted to pediatric patients..$^{15}$

Some patients present with congenital anomalies concomitant to DBA, such as craniofacial, cardiac, genitourinary, and upper limb inborn deformities. ${ }^{1,2,4-7,16-19}$ Low birth weight and growth retardation are also reported. With no specific role described so far, $, 1,7,8$ ribosomal protein S19 (RP S19) was the first mutated gene to be linked to DBA., ${ }^{1,6,8,14,18-21}$ It has been proposed that DBA results from haplodeficiency in patients presenting with this mutation. ${ }^{1,4,18,21,22}$ Several other mutated ribosomal proteins have been identified, indicating that DBA is an outcome of defects in biogenesis or ribosomal function. $1,3,4,8,23$

The common characteristics of DBA include significant anemia early in life, though with normal neutrophil and platelet counts. ${ }^{9,13,14}$ Thrombocytopenia and neutropenia may occur, ${ }^{9,13}$ and reticulocytopenia, normal macrocytosis, and cellularity of the bone marrow should also be considered besides reduction of erythroid progenitors. ${ }^{1,15,16,24}$
Corticosteroid therapy is the main treatment approach to DBA, since a positive response is observed in most cases, ${ }^{1,5,6}$ and the disease remains controlled in others for a considerable length of time. ${ }^{7}$ Nevertheless, approximately $50 \%$ of patients discontinue the treatment due to the loss of clinical efficacy or to secondary effects. ${ }^{2,6}$ Between $15 \%$ to $20 \%$ of patients exhibit resistance to corticoid therapy at diagnosis of DBA. ${ }^{20}$ Blood transfusion is prescribed during diagnosis and in the chronic manifestations of DBA, when the patient ceases to respond to corticosteroid therapy. ${ }^{1,2,23}$ Research has described the curative role of hematopoietic stem cell transplantation (HSCT) in DBA. ., $, 16,19,23,25$

The clinical evolution of DBA patients is unpredictable, and complications are usually severe, most of which are associated with treatment due to the chronic use of corticosteroids, increased iron levels, infections, and side effects associated with transplantation. ${ }^{16,18}$ In addition, DBA patients are at higher risk of acquiring malignant diseases, solid tumors (bone, breast, and digestive tract cancers), and hematological neoplasias. ${ }^{1,16}$

Neutropenia is characterized by low total neutrophil counts. In peripheral blood, normal neutrophil counts stand between 3,000 and 6,000 cells $/ \mathrm{mm}^{3}$, and values below 500 cells $/ \mathrm{mm}^{3}$ indicate severe neutropenia. ${ }^{26-28}$ The occurrence of oral lesions
Corresponding Author: Dra. Maria Cristina Munerato, Faculdade de Odontologia - UFRGS, Rua Ramiro Barcelos, 2492, Departamento de Odontologia Conservadora (DOC), Porto Alegre, RS. Brazil. 90035-003, Tel: +55 5I 33598248,

Fax: +55 5 I 33085005, Email: mcmunerato@gmail.com
Received: September 20, 2015

Ist Revision: November 30, 2015

2nd Revision: December 18, 2015

Accepted: December 23, 2015 
in DBA has been correlated with the severity of neutropenia and thrombocytopenia. ${ }^{26}$ Fever of unknown origin may be reported, and oral manifestations usually start with the emergence of petechiae that, in more severe cases, evolve to gingivitis, necrotic lesions in the palate and gingivae, stomatitis, cellulitis, abscesses, and other severe systemic infections. ${ }^{17,26,28,29}$ Since the inflammatory response in patients with neutropenia is less intense, signs of infection such as thermal discomfort, blushing, and edema are less evident or may not even be present at all. After agranulocytosis that may stretch from a few days to a few weeks, patients report fever, fits of cold, shivering, general indisposition, and, in some cases, considerable weariness. Pulmonary and oropharyngeal infection foci are also observed. Additionally, urinary tract infections and other signs are also reported. ${ }^{27,28}$

This study presents a case of DBA in a patient with oral mucosa lesions due to secondary neutropenia who underwent treatment in the Stomatology Unit, Hospital de Clínicas de Porto Alegre (HCPA), Rio Grande do Sul, Brazil.

\section{Case Presentation}

An African American woman, age 20 years, with DBA presented to the Emergency Service Department of HCPA complaining of fever and painful ulcers on the oral mucosa, as well as urinary infection, severe neutropenia, and severe chronic anemia that did not respond properly to previous treatment. Stomatological examination was requested by the medical team as a tool towards diagnosis and treatment of the oral lesions.

The patient's clinical history included severe hyporesponsive anemia since childhood, splenectomy at the age of 10 years, DBA diagnosis confirmed at the age of 17 years after bone marrow biopsy, and Cushing Syndrome due to chronic use of corticosteroids. Physical examination did not reveal any change associated with DBA as described in the literature, such as craniofacial, urogenital, cardiac, musculoskeletal, and neuromotor anomalies, in addition to changes in upper limbs (fingers) that may be present in as many as $25 \%$ of patients with DBA.

Clinical examination of the oral cavity indicated the presence of two painful $8 \mathrm{~mm}$ crateriform ulcers covered with irregularly shaped pale pseudomembrane on the lower lip labial mucosa, to the left of the median line (figures 1A \& 1B) that bled upon handling. On the right edge of the tongue there was a flat-edged, well-outlined, shallow $5 \mathrm{~mm} \times 8 \mathrm{~mm}$ ulcer covered with irregularly shaped pale pseudomembrane that was painful but not hemorrhagic (figure 1C).

The patient displayed good oral health conditions, all permanent teeth were healthy and erupted, with no anomalies or sign of periodontal disease. The patient undergoes clinical odontological follow-up outside HCPA by a dental surgeon at variable intervals. Currently the patient is under orthodontic treatment.
The initial diagnostic hypothesis was of ulcers associated with neutropenia, more severe by secondary contamination and reduced inflammatory response. Diagnosis was made clinically, with no need for biopsy or histopathological investigation, at that time.

During the 18 days the patient was hospitalized, the urinary infection was treated with intravenous antibiotics (cefepime 2 grams every 8 hours for 5 days, and vancomycin 1.5 grams every 12 hours for 7 days) and oral antibiotics (sulfamethoxazole and trimethoprim $960 \mathrm{mg}$ every 12 hours for 14 days). Severe and persistent anemia with neutropenia was treated with blood transfusions and granulocyte colonystimulating factor (G-CSF, filgrastim) upon hemodynamic stabilization. This treatment was defined by the medical team.

The stomatological treatment was carried out using three approaches: (a) daily low-frequency laser therapy to reduce pain and stimulate cellular renovation and lesion healing; (b) chlorhexidine $0.12 \%$ mouthwashes twice a day to chemically control biofilm formation and therefore avoid secondary contamination of oral ulcers, which would delay healing; and (c) topical lidocaine $10 \%$ spray (to make eating more comfortable). The patient was examined daily by the stomatology team as a means to monitor the healing of oral lesions, which was reached 10 days after the beginning of laser treatment. Hemodynamic stabilization was observed at the same time, confirming the preliminary hypothesis of neutropenic ulcers (figures 1D, 1E, \& 1F).

If total reparation of oral lesions had not been observed, partial biopsy of one ulcer would have been prescribed. In this case, differential diagnosis would be reached based on infectious diseases and autoimmune mucocutaneous diseases that may elicit multiple oral mucosa lesions. These diseases would include syphilis, deep mycoses, lichen planus, pemphigus, and pemphigoids.

Currently the patient is being followed clinically, polytransfused, and receiving concentrated adult erythrocyte suspensions once per week. Iron overdose is also being controlled. Hemoglobin and erythrocyte counts remain extremely low despite treatment with erythropoietin, prednisone, and cyclosporine A. However, no new neutropenia has emerged, and no other oral lesions have been observed.

\section{Discussion}

DBA was first described by Josephs in $1936 .^{30}$ The disease often manifests between the third month and the second year of life, ${ }^{16}$ though it has been diagnosed in patients from other age groups ${ }^{15}$ Recent data show that approximately $40 \%$ to $50 \%$ of DBA cases are inherited in an autosomal dominant pattern, ${ }^{4,20,31}$ though gene transmission is unpredictable. ${ }^{17}$ The hereditary character of the disease underlines the importance 

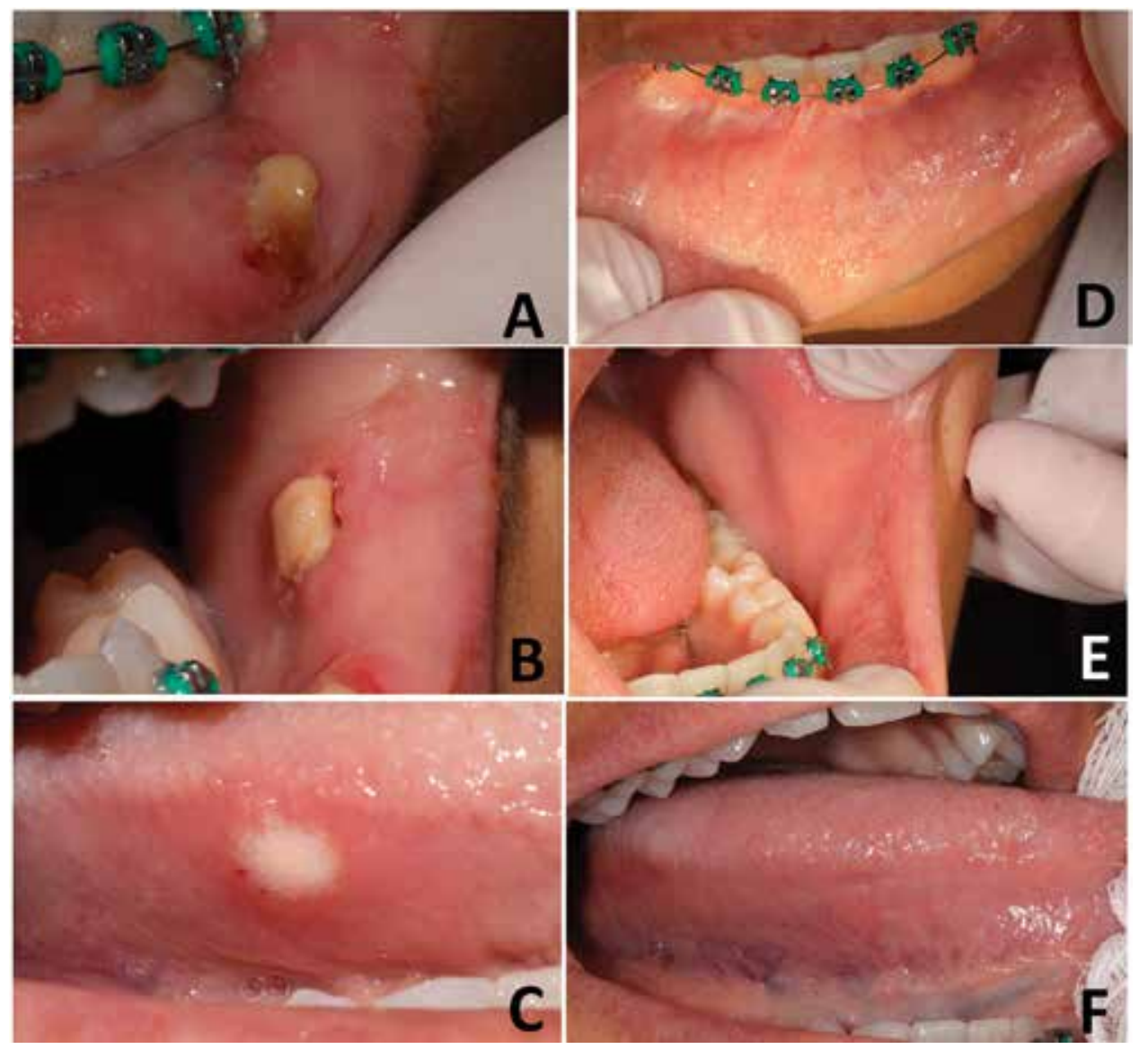

Figure 1. Ulcers in the oral mucosa observed during neutropenia (A, B, and $C$ ), and total remission after 10 days, when hemodynamic balance was achieved (D, E, and F).

of a genetic counseling., ${ }^{4,32}$ Prevalence of DBA is at one case in every 100,000 to 200,000 inhabitants, with no ethnic or gender predisposition. ${ }^{1,5,8,19,21}$

Few studies have investigated the diagnosis of DBA in adult patients. ${ }^{33}$ In the present case report, the patient did not present the physical anomalies associated with DBA, which make early diagnosis more difficult. Although the patient exhibited severe anemia since the very first months of life, a conclusive diagnosis was not reached until she was age 17 years. Another critical issue lies in the fact that the patient was born and lived in a small town in the state of Rio Grande do Sul, Brazil, where specialized health services are not available to diagnose rare hematological diseases.

DBA has traditionally been described as a pure red cell aplasia (PRCA), with defects restricted to the erythroid linage, ${ }^{9}$ though the first reports described mild neutropenia and thrombocytopenia. A case series study that analyzed the evolution of DBA in 28 patients pointed to a statistically significant number of individuals who were unresponsive to corticosteroid therapy, and who developed thrombocytopenia and/or neutropenia with increasing age. ${ }^{9}$ Eleven patients were followed for more than 15 years, of which six presented with some degree of cytopenia, and one died from neutropenic sepsis. ${ }^{9}$ Lower counts of the three blood cell lines were exhibited in $75 \%$ of patients. Neutropenia was present in four individuals, of which three also manifested thrombocytopenia. ${ }^{9}$

Agranulocytosis may also be induced by medicinal drugs, culminating in a relevant and sudden hematological condition that emerges as a result of an idiosyncratic reaction to a given medicament. The mechanism behind neutropenia has not been completely elucidated, though the condition has been reported to occur due to prolonged treatment with a specific medicinal drug, metabolic conditions, lowered neutrophil production in the bone marrow, or high plasma levels of a drug. ${ }^{27}$

In the present report, the patient manifested severe febrile neutropenia at the age of 19 years, requiring admission to a hospital to treat opportunistic infections and ensure hemodynamic stabilization. Among the co-morbidities associated with febrile neutropenia, the literature cites urinary tract infections and aggressive, painful ulcers on the oral cavity that are morphologically similar to those described in cyclic neutropenia patients. ${ }^{17}$ Apart from presenting with DBA, the patient was given prednisone, cyclosporine A, deferasirox, and erythropoietin chronically. It should be emphasized that the literature reports the death of a girl, age 12 years, from agranulocytosis induced by deferiprone, a chelating agent..$^{20,23}$ However, although deferasirox is a 
chelating agent also, it is efficient and safe for controlling iron levels $\mathrm{s}^{24,34,35,36}$ and has been linked to agranulocytosis only when administered at high doses. ${ }^{16}$

Patients with febrile neutropenia require long-term, broadspectrum antibiotic therapy, depending on the associated etiologic agent. ${ }^{27-29}$ Severe cases demand hospital admission with isolation of the patient and strict antiseptic measures, apart from specific care in terms of diet and oral health. ${ }^{27}$ In the present case report, the patient exhibited severe neutropenia, urinary infection, and painful crateriform lesions on the oral mucosa. In such a scenario, the patient was admitted to the hospital to receive the appropriate intravenous antibiotic regimen to eliminate the infection in the urinary tract. Moreover, the period of hospitalization also allowed for administration of filgastrim to quickly increase neutrophil counts and to conduct blood transfusions until hemodynamic balance was reached. ${ }^{27}$ Since the oral lesions would have healed spontaneously with recovery of the hematological condition, our approach was directed to palliative care. The mouth was rinsed with aqueous chlorhexidine $0.12 \%$ solution every 12 hours to prevent any secondary infection of the lesions. Also, lidocaine 10\% topical spray applications were prescribed to mitigate symptoms, and daily laser therapy sessions were prescribed as adjuvant analgesic therapy and to speed up healing.

The specific approaches prescribed to increase absolute neutrophil counts include corticosteroid therapies as well as the prescription of intravenous immunoglobulins and plasmapheresis, especially for patients with severe infectious complications. ${ }^{27}$ Myeloid lineage growth factors such as G-CSF (filgastrim/lenograstim) and GM-CSF (molgramostim) stimulate the production of neutrophil precursor cells and activate the phagocytic and cytotoxic functions of mature neutrophils. Among the beneficial effects of G-CSF are increased neutrophil counts, shorter neutropenia times, and lower infection rates, apart from the possibility of reducing hospital admissions. ${ }^{28,37}$ Filgastrim induces a fast increase in circulating neutrophil counts, eliciting the remission of oral lesions caused by neutropenia. ${ }^{28}$

A case report of two patients with $\mathrm{DBA}^{13}$ described the issues affecting oral health status in this disease. Although a considerable build-up of biofilm was reported due to poor oral hygiene, the other periodontal conditions of these patients were considered normal. Few studies have described oral findings in DBA patients. The oral and dental conditions reported include severe gingivitis, multiple caries lesions, impacted third molars, and obliteration of the crown pulp chamber. ${ }^{13}$ In the present case report, the patient presented with good mechanical control of biofilm formation, with preserved teeth and no evidence of periodontal disease. Currently she is under orthodontic treatment.
Corticosteroid regimens, chronic blood transfusions, and HSCT are the current treatments of choice for DBA. ${ }^{3,23}$ According to the USA DBA databank, overall survival is $75.1 \%$ at the age of 40 years, which requires concerted efforts in the search for new alternatives for increasing survival rates at the same time that any complications associated with these therapies are mitigated. $3,18,23$

HSCT is prescribed when the patient requires constant blood transfusion, and it is the only definitive treatment for hematological manifestations of DBA. The 2008 Consensus Guidelines, which established the diagnosis and treatment criteria for DBA that are currently adopted, recommends that HSCT be prescribed as the treatment of choice for patients of up to age 10 years. For older patients, it is recommended only to treat cytopenia that affects lineages other than the erythroid, myelodysplasia, and acute myeloid leukemia. ${ }^{23}$ Promising results have been described for bone marrow transplants from consanguineous donors, though an in-depth evaluation of possibilities is required in light of the risk of DBA in the family and the complex diagnosis of the condition. ${ }^{1,7,8,16,22}$ Evaluation of the patient by the medical team indicated that transplantation was not appropriate at that moment. The literature indicates that HSCT should be carried out only if remission after other therapies is not observed. ${ }^{6,14,22}$ However, the remission mechanism of DBA remains unclear. ${ }^{6}$ A previous study concluded that HSCT conducted before multiple transfusions reduces iron levels, improving neutrophil and platelet recovery after transplantation. ${ }^{22}$

\section{Conclusion}

Descriptions of oral manifestations associated with DBA in the literature are rare. Based on this case report and on the literature review, it is possible to conclude that the oral ulcers observed had no direct association with DBA, but with neutropenia, which is a frequent co-morbidity factor in these patients.

The role of a stomatologist in the transdisciplinary team treating patients with DBA is justified in light of the importance of accompanying oral health conditions, in addition to the diagnosis and treatment of oral lesions. Whenever necessary, a patient with DBA may be referred for conventional less invasive dental treatment in specific oral health units.

Oral lesions in patients with DBA may be associated with hemodynamic imbalance, which should be seen as an early clinical warning. Two reasons explain the importance of stomatological follow-up: (a) patients with DBA are under chronic treatment with corticosteroids and, therefore, are more susceptible to infections in the oral cavity; and (b) these patients are more inclined to develop solid malignant neoplasias that may also manifest in the oral cavity. 


\section{References}

1. Vlachos A, Ball S, Dahl N, Alter BP, Sheth S, Ramenghi U, Meerpohl J, Karlsson S, Liu JM, Leblanc T, Paley C, Kang EM, Leder EJ, Atsidaftos E, Shimamura A, Bessler M, Glader B, Lipton JM; Participants of Sixth Annual Daniella Maria Arturi International Consensus Conference. Diagnosing and treating Diamond Blackfan anaemia: results of an international clinical consensus conference. Br J Haematol 2008;142:859-876

2. Beshlawy AEl, Ibrahim IY, Rizk S, Eid K. Study of 22 Egyptian patients With Diamond-Blackfan Anemia, corticosteroids, and cyclosporin therapy results. Pediatrics 2002;110;e44.

3. Chen YM, Ruan M, Wang YQ, Zhang L, Liu TF, Zhu XF. [Analysis of clinical characteristics in 45 cases of DiamondBlackfan anemia]. [Article in Chinese] Zhongguo Shi Yan Xue Ye Xue Za Zhi. [Journal of Experimental Hematology] 2012;20(3):646-649.

4. Chung NG, Kim M. Current insights into inherited bone marrow failure syndromes. Korean J Pediatr 2014;57:337-344.

5. Ball SE, McGuckin CP, Jenkins G, Gordon-Smith EC. DiamondBlackfan anaemia in the U.K.: analysis of 80 cases from a 20-year birth cohort. Br J Haematol 1996;94:645653.

6. Sjögren SE, Flygare J. Progress towards mechanism-based treatment for Diamond-Blackfan anemia. ScientificWorld Journal 2012;184362.

7. Vilan A, Cunha J, Brandao C, Cleto E. Anemia de DiamondBlackfan. Acta Pediatr Port 2008:39:72-74.

8. Ohga S, Mugishima H, Ohara A, Kojima S, Fujisawa K, Yagi K, Higashigawa M, Tsukimoto I; Aplastic Anemia Committee Japanese Society of Pediatric Hematology. DiamondBlackfan anemia in Japan: clinical outcomes of prednisolone therapy and hematopoietic stem cell transplantation. Int J Hematol 2004;79:22-30.

9. Giri N, Kang E, Tisdale JF, Follman D, Rivera M, Schwartz GN, Kim S, Young NS, Rick ME, Dunbar CE. Clinical and laboratory evidence for a trilineage haematopoietic defect in patients with refractory Diamond-Blackfan anaemia. Br J Haematol 2000;108:167-175.

10. Lipton JM, Kudisch M, Gross R, Nathan DG. Defective erythroid progenitor differentiation system in congenital hypoplastic (Diamond-Blackfan) anemia. Blood 1986;67:962968.

11. Ohene-Abuakwa Y, Orfali KA, Marius C, Ball SE. Two-phase culture in Diamond-Blackfan anemia: localization of erythroid defect. Blood 2005; 105:838-846.

12. Perdahl EB, Naprstek BL, Wallace WC, Lipton JM. Erythroid failure in Diamond-Blackfan anemia is characterized by apoptosis. Blood 1994;83:645-650.

13. Ozden FO, Gunduz K, Ozden B, Isci KD, Fisgin T. Oral and dental manifestations of diamond-blackfan anemia: case reports. Eur J Dent 2011;5:344-348.

14. Djaldetti M, Blay A, Bergman M, Salman H, Bessler H. Pure red cell aplasia--a rare disease with multiple causes. Biomed Pharmacother 2003;57:326-332.

15. Alter BP. Diagnosis, genetics, and management of inherited bone marrow failure syndromes. Hematology Am Soc Hematol Educ Program 2007:29-39.

16. Costa L, Moniz H, Simansour M,Tchernia G, Mohandas N, Leblanc T. Diamond-Blackfan anemia, ribosome and erythropoiesis. Transfus Clin Biol 2010;17:112-119.

17. Rezaei N, Farhoudi A, Pourpak Z, Aghamohammadi A, Ramyar A, Moin M, Gharagozlou M, Movahedi M, Mohammadpour B, Mirsaeid Ghazi B, Izadyar M, Mahmoudi M. Clinical and laboratory findings in Iranian Children with cyclic neutropenia. Iran J Allergy Asthma Immunol 2004;3:37-40.

18. Ball S. Diamond Blackfan anemia. Hematology Am Soc Hematol Educ Program 2011;487-491.
19. Ramenghi U, Garelli E, Valtolina S, Campagnoli MF, Timeus F, Crescenzio N, Mair M, Varotto S, Avanzo MD, Nobili B, Massolo F, Mori PG, Locatelli F, Gustavsson P, Dahl N, Dianzani I. Diamond-Blackfan anaemia in the Italian population. Br J Haematol 1999;104:841-848.

20. Henter JI, Karlén J. Fatal agranulocytosis after deferiprone therapy in a child with Diamond-Blackfan anemia. Blood 2007; 109:5157-5159.

21. Willig TN, Draptchinskaia N, Dianzani I, Ball S, Niemeyer C, Ramenghi U, Orfali K, Gustavsson P, Garelli E, Brusco A, Tiemann C, Pérignon JL, Bouchier C, Cicchiello L, Dahl N, Mohandas N, TcherniaG. Mutations in ribosomal protein S19 gene and diamond-blackfan anemia: wide variations in phenotypic expression. Blood 1999;94:4294-4306.

22. Roy V, Pérez WS, Eapen M, Marsh JC, Pasquini M, Pasquini R, Mustafa MM, Bredeson CN; Non-Malignant Marrow Disorders Working Committee of the International Bone Marrow Transplant Registry. Bone marrow transplantation for diamond-blackfan anemia. Biol Blood Marrow Transplant 2005:11:600-608.

23. Narla A, Vlachos A, Nathan DG. Diamond Blackfan anemia treatment: past, present, and future. Semin Hematol 2011;48:117-123.

24. Cassinerio E, Orofino N, Roghi A, Duca L, Poggiali E, Fraquelli M, Zanaboni L, Cappellini MD. Combination of deferasirox and deferoxamine in clinical practice: an alternative scheme of chelation in thalassemia major patients. Blood Cells Mol Dis 2014;53:164-167.

25. Vlachos A, Federman N, Reyes-Haley C, Abramson J, Lipton JM. Hematopoietic stem cell transplantation for Diamond Blackfan anemia: a report from the Diamond Blackfan Anemia Registry. Bone Marrow Transplant 2001;27:381-386.

26. Poffo DJ. Universidade Federal de Santa Catarina. Curso de Graduação em Odontologia. Úlceras bucais relacionadas às principais desordens sistêmicas. 2012. Available at: https:// repositorio.ufsc.br/bitstream/ handle/123456789/103611/D\%C3\%A9bora\%20Julite\%20 Poffo.pdf?sequence=. Accessed March 10, 2015.

27. Alonso RG, Andrade CRG, Guimerá VP, Puente IM. Agranulocitosis inducida por medicamentos Terapéutica 2004; Jan16(1518) 23-29.

28. Matarasso S, Daniele V, Iorio Siciliano V, Mignogna MD, Andreuccetti G, Cafiero C. The effect of recombinant granulocyte colony-stimulating factor on oral and periodontal manifestations in a patient with cyclic neutropenia: a case report. Int J Dent 2009;2009:654239.

29. Jabbari H, Payvarmehr F, SeyedAlinaghi S, Roosta N. A case report of cyclic neutropenia associated with pyoderma gangrenosum. Acta Med Iran 2011;49:763-765.

30. Josef HW. Anaemia of infancy and early childhood. Medicine 1936:15:307-451

31. Orfali KA, Ohene-Abuakwa Y, Ball SE. Diamond Blackfan anaemia in the UK: clinical and genetic heterogeneity. Br J Haematol 2004; 125:243-252.

32. Tamary H, Nishri D, Yacobovich J, Zilber R, Dgany O, Krasnov T, Aviner S, Stepensky P, Ravel-Vilk S, Bitan M, Kaplinsky C, Ben Barak A, Elhasid R, Kapelusnik J, Koren A, Levin C, Attias D, Laor R, Yaniv I, Rosenberg PS, Alter BP. Frequency and natural history of inherited bone marrow failure syndromes: the Israeli Inherited Bone Marrow Failure Registry. Haematologica 2010;95:1300-1307.

33. Balaban EP, Buchanan GR, Graham M, Frenkel EP. DiamondBlackfan syndrome in adult patients. Am J Med 1985;78:533538. 
34. Cappellini MD, Pattoneri P. Oral iron chelators. Annu Rev Med 2009;60:25-38.

35. Cappellini MD, Piga A. Current status in iron chelation in hemoglobinopathies. Curr Mol Med 2008;8:663-674.

36. Cermak J, Jonasova A, Vondrakova J,Cervinek L, Belohlavkova P, Neuwirtova R. A comparative study of deferasirox and deferiprone in the treatment of iron overload in patients with myelodysplastic syndromes. Leuk Res 2013;37:1612-1615.

37. Ministério Da Saúde. Secretaria De Assistência À Saúde.

Portaria SAS/MS n ${ }^{\circ} 862,04$ de novembro de 2002.

Departamento Nacional de Auditoria do SUS. Coordenação de Sistemas de Informação Sistema Único de Saúde Legislação Federal. DO 214, 5/11/02. Available at http://sna. saude.gov.br/legisla/legisla/informes/SAS_P862_02informes. doc. Accessed March 10, 2015.

\section{Author Affiliations}

Rita Fabiane Teixeira Gomes* and Maria Cristina

Munerato, $P h D$, * *

*Dentistry School, Federal University of Rio Grande do Sul (UFRGS), Porto Alegre, Brazil

${ }^{+}$Associate Professor, Dentistry School, Federal University of Rio Grande de Sul (UFRGS), Porto Alegre, Brazil

* Stomatology Unit, Hospital de Clínicas de Porto Alegre

(HCPA), Porto Alegre, Brazil 